PROCEEDINGS OF THE

AMERICAN MATHEMATICAL SOCIETY

Volume 140, Number 11, November 2012, Pages 3853-3859

S 0002-9939(2012)11225-0

Article electronically published on March 5, 2012

\title{
UNIQUENESS OF CRITICAL TRAVELING WAVES FOR NONLOCAL LATTICE EQUATIONS WITH DELAYS
}

\author{
ZHI-XIAN YU
}

(Communicated by Yingfei Yi)

\begin{abstract}
In this paper, we investigate uniqueness (up to translation) of critical traveling waves for delayed lattice equations with monotone or nonmonotone birth functions. Our method requires finding exactly a priori asymptotic behavior of the critical traveling wave. This we accomplish with the help of Ikehara's Theorem.
\end{abstract}

\section{INTRODUCTION}

Consider the following system of differential equations on lattice $\mathbb{Z}$ :

$(1.1)$

$$
u_{n}^{\prime}(t)=D\left[u_{n+1}(t)+u_{n-1}(t)-2 u_{n}(t)\right]-d u_{n}(t)+\sum_{j \in \mathbb{Z}} \beta(n-j) b\left(u_{j}(t-r)\right), n \in \mathbb{Z},
$$

which describes the distribution of matured population of a single species over patch $\mathbb{Z}$; see Weng et al. [14]. Here $\beta(-j)=\beta(j) \geq 0$ with $\sum_{j \in \mathbb{Z}} \beta(j)=1$, $b(0)=d K-b(K)=0$ for some $K>0$. A traveling wave of (1.1) with speed $c$ is a nonnegative bounded solution of the form $u_{n}(t)=\phi(n+c t)$ satisfying $\phi(-\infty)=0$ and $\liminf \operatorname{in}_{\xi \rightarrow+\infty} \phi(\xi)>0$. Substituting $u_{n}(t)=\phi(n+c t)$ into (1.1), we have the following wave profile equation:

$$
c \phi^{\prime}(\xi)=D[\phi(\xi+1)+\phi(\xi-1)-2 \phi(\xi)]-d \phi(\xi)+\sum_{j \in \mathbb{Z}} \beta(j) b(\phi(\xi-j-c r)) .
$$

In the case where $b(w)$ is monotone in $w \in[0, K]$, Weng et al. 14 obtained the spreading speed $c_{*}$ and a monotone traveling wave for the wave speed $c \geq c_{*}$. When $c>c_{*}$, Ma and Zou 12 established the uniqueness (up to translation) and stability of traveling waves for the local case (i.e. $\beta(j)=0$ for all $j \neq 0$ ) of equation (1.1) with monotone birth functions. For the nonmonotone case, Ma et al. 13. established the spreading speed $c_{*}$ and Ma [10] obtained the existence of traveling waves for the nonlocal lattice equation by the method used in reaction diffusion equation (see Remark 1.4 and Theorem 1.2 in [10]). Recently, for (1.1) with nonmonotone birth functions, Fang et al. [8] further studied the spreading speed $c_{*}$ by the comparison argument and the fluctuation method, and the existence of traveling waves for $c \geq c_{*}$ by Schauder's fixed point theorem.

Received by the editors November 21, 2010 and, in revised form, April 21, 2011 and April 22, 2011.

2010 Mathematics Subject Classification. Primary 34K31.

The work of the author was supported by the Natural Science Foundation of China (No. 11101282).

(C)2012 American Mathematical Society Reverts to public domain 28 years from publication 
More recently, the authors of [9] established the uniqueness of traveling waves of (1.1) for $c>c_{*}$ without assuming that wave profile is monotone, which was based on exactly a priori asymptotic behavior of the wave profile. Although this was accomplished by developing a structure theorem of entire solutions to a class of linear integro-differential equations, the use of the theorem (i.e. Theorem 2.1 in 9]) has a disadvantage; that is, the conclusion holds only when the characteristic equation has different eigenvalues in the belt region. Note that the characteristic equation $\Delta(c, \lambda)=0$ of the wave profile equation (1.2) has double roots for $c=c_{*}$, and so Theorem 2.1 in [9] could not be applied. Our object is to give a proof about the uniqueness of traveling waves of (1.1) for $c=c_{*}$ with the help of Ikehara's Theorem. This technique was also used in 2] to prove the uniqueness of traveling waves for some monostable integrodifferential equations. To my knowledge, no result on uniqueness of critical traveling waves for delayed lattice systems has been reported. Other methods to prove uniqueness of noncritical traveling waves for other types of evolution systems can be found in [1, 3, 4, 5, 6, 7, 11, 12]. In [8], it is also shown that for (1.1), the minimal wave speed $c_{*}$ coincides with the spreading speed and the linear determinacy holds for (1.1), meaning that $c_{*}$ is fully determined by the characteristic equation of the linearization of (1.1) at the trivial equilibrium.

\section{UNIQUENESS OF CRITICAL TRAVELING WAVES}

In this section, we show the uniqueness of traveling waves of (1.1) for $c=c_{*}$. In order to accomplish this, we find exactly a priori asymptotic behavior of the critical traveling wave with the help of Ikehara's Theorem. Throughout this section, we assume that $\phi\left(n+c_{*} t\right)$ is a nonnegative bounded critical traveling wave (wave shape is monotone or nonmonotone) of (1.1) with $\phi(-\infty)=0$ and $\liminf _{\xi \rightarrow+\infty} \phi(\xi)>0$.

Assume that the function $b(u)$ is differentiable at $u=0$. Define the characteristic equation

$$
\Delta(c, \lambda):=c \lambda-D\left(e^{\lambda}+e^{-\lambda}-2\right)+d-b^{\prime}(0) \sum_{j \in \mathbb{Z}} \beta(j) e^{-\lambda(j+c r)},
$$

where $c$ is regarded as a parameter. We make the following assumptions on functions $\beta$ and $b$ :

(H1) $\beta(j)=\beta(-j) \geq 0$ for all $j \in \mathbb{Z} ; \sum_{j \in \mathbb{Z}} \beta(j)=1$; there exists $\lambda^{\sharp}>0$ such that $\chi(\lambda)=: \sum_{j \in \mathbb{Z}} \beta(j) e^{-\lambda j}$ is convergent when $\lambda \in\left[0, \lambda^{\sharp}\right)$ and $\lim _{\lambda \rightarrow \lambda^{\sharp}} \chi(\lambda)=$ $+\infty$, where $\lambda^{\sharp}$ may be $+\infty$.

(H2) $b$ is continuous from $\mathbb{R}^{+}$to $\mathbb{R}^{+}$with $b^{\prime}(0)>d$; there exist $a>0, \delta>0$ and $\sigma>1$ such that $b(u) \geq b^{\prime}(0) u-a u^{\sigma}$ for all $u \in[0, \delta]$.

(H3) For all $u_{1}, u_{2} \geq 0,\left|b\left(u_{1}\right)-b\left(u_{2}\right)\right| \leq b^{\prime}(0)\left|u_{1}-u_{2}\right|$.

From Lemma 3.1 in 9 ] and Lemma 2.2 in [13, we have

Lemma 2.1. Assume that $(\mathrm{H} 1)$ holds and $b^{\prime}(0)>d$. Then, there exists a unique pair of $c_{*}>0$ and $\lambda_{*}>0$ such that the following assertions hold:

(i) $\Delta\left(c_{*}, \lambda_{*}\right)=\left.0 \cdot \frac{\partial \Delta(c, \lambda)}{\partial \lambda}\right|_{c=c_{*}, \lambda=\lambda_{*}}=0$.

(ii) For any $c>c_{*}, \Delta(c, \lambda)=0$ has exactly two positive roots $\lambda_{1}<\lambda_{2}<\lambda^{\sharp}$ and $\Delta(c, \lambda)>0$ for any $\lambda \in\left(\lambda_{1}, \lambda_{2}\right)$.

(iii) $\Delta\left(c_{*}, \lambda\right)$ does not have any zeros with $\Re \lambda=\lambda_{*}$ other than $\lambda=\lambda_{*}$.

We recall a version of Ikehara's Theorem. 
Lemma 2.2 ([2], Proposition 2.3). Let $F(\lambda)=\int_{0}^{+\infty} u(x) e^{-\lambda x} d x$, with u being a positive decreasing function. Assume that $F(\lambda)$ has the representation

$$
F(\lambda)=\frac{h(\lambda)}{(\lambda+\alpha)^{k+1}},
$$

where $k>-1$ and $h$ is analytic in the strip $-\alpha \leq \operatorname{Re} \lambda<0$. Then

$$
\lim _{x \rightarrow+\infty} \frac{u(x)}{x^{k} e^{-\alpha x}}=\frac{h(-\alpha)}{\Gamma(\alpha+1)}>0 .
$$

Theorem 2.1 (Asymptotic behavior). Assume that (H1)-(H2) hold. Let $\phi\left(n+c_{*} t\right)$ be a traveling wave of (1.1) with $\phi(-\infty)=0$. Then

$$
\lim _{\xi \rightarrow-\infty} \frac{\phi(\xi)}{|\xi| e^{\lambda_{*} \xi}} \text { exists. }
$$

Proof. We claim that $\phi$ is positive. Suppose to the contrary that there exists $\xi_{1} \in \mathbb{R}$ such that $\phi\left(\xi_{1}\right)=0$. Since $\phi$ is a nonnegative bounded traveling wave with $\phi(-\infty)=0$ and $\liminf _{\xi \rightarrow+\infty} \phi(\xi)>0, \xi_{0}:=\sup \{\xi \in \mathbb{R} \mid \phi(\xi)=0\}$ is well defined and $\phi\left(\xi_{0}\right)=\phi^{\prime}\left(\xi_{0}\right)=0$. Thus,

$$
\begin{aligned}
0=c \phi^{\prime}\left(\xi_{0}\right) & =D\left[\phi\left(\xi_{0}+1\right)+\phi\left(\xi_{0}-1\right)-2 \phi\left(\xi_{0}\right)\right]-d \phi\left(\xi_{0}\right)+\sum_{j \in \mathbb{Z}} \beta(j) b\left(\phi\left(\xi_{0}-j-c r\right)\right) \\
& \geq D \phi\left(\xi_{0}+1\right)+D \phi\left(\xi_{0}-1\right) \geq 0,
\end{aligned}
$$

which implies that $\phi\left(\xi_{0}+1\right)=0$. This contradicts the definition of $\xi_{0}$.

Similar to the proof of Lemma 3.4 in [9], the two-sided Laplace transform of $\phi$ by

$$
L(\lambda) \equiv \int_{\mathbb{R}} \phi(\theta) e^{-\lambda \theta} d \theta
$$

is analytic for any $\Re \lambda \in\left(0, \lambda_{*}\right)$ and $L(\lambda)$ has a singularity at $\lambda=\lambda_{*}$. Since

$$
\int_{\mathbb{R}} e^{-\lambda \theta}[\phi(\theta+1)+\phi(\theta-1)-2 \phi(\theta)] d \theta=L(\lambda)\left(e^{\lambda}+e^{-\lambda}-2\right)
$$

and

$$
\begin{aligned}
& c_{*} \phi^{\prime}(\xi)-D[\phi(\xi+1)+\phi(\xi-1)-2 \phi(\xi)]+d \phi(\xi)-b^{\prime}(0) \sum_{j \in \mathbb{Z}} \beta(j) \phi\left(\xi-j-c_{*} r\right) \\
= & -b^{\prime}(0) \sum_{j \in \mathbb{Z}} \beta(j) \phi\left(\xi-j-c_{*} r\right)+\sum_{j \in \mathbb{Z}} \beta(j) b\left(\phi\left(\xi-j-c_{*} r\right)\right):=R(\phi)(\xi),
\end{aligned}
$$

it follows that

$$
\Delta\left(c_{*}, \lambda\right) L(\lambda)=\int_{-\infty}^{\infty} e^{-\lambda \theta} R(\phi)(\theta) d \theta
$$

Similarly to the argument in the proof of Lemma 3.4 in 9 , there exists $\eta>0$ such that the right hand side of (2.2) is analytic for $\lambda \in\left(0, \lambda_{*}+\eta\right)$. We rewrite (2.2) as

$$
\int_{-\infty}^{0} \phi(\theta) e^{-\lambda \theta} d \theta=\frac{\int_{\mathbb{R}} e^{-\lambda \theta} R(\phi)(\theta) d \theta}{\Delta\left(c_{*}, \lambda\right)}-\int_{0}^{\infty} \phi(\theta) e^{-\lambda \theta} d \theta .
$$

Note that $\int_{0}^{\infty} \phi(\theta) e^{-\lambda \theta} d \theta$ is analytic for $\Re \lambda>0$. Also, by Lemma 2.1 (iii), $\Delta\left(c_{*}, \lambda\right)=0$ does not have any zero with $\Re \lambda=\lambda_{*}$ other than $\lambda=\lambda_{*}$. 
Assume that $\phi(\xi)$ is increasing for large $-\xi>0$. Then we can choose a translation of $\phi$ such that it is increasing for $\xi<0$. Letting $u(\xi)=\phi(-\xi)$ and $T(u)(\xi):=$ $-b^{\prime}(0) \sum_{j \in \mathbb{Z}} \beta(j) u\left(\xi+j+c_{*} r\right)+\sum_{j \in \mathbb{Z}} \beta(j) b\left(u\left(\xi+j+c_{*} r\right)\right)$, it is clear that $u(\xi)$ is decreasing $\xi>0$ and

$$
\int_{0}^{\infty} u(\theta) e^{\lambda \theta} d \theta=\frac{\int_{\mathbb{R}} e^{\lambda \theta} T(u)(\theta) d \theta}{\Delta\left(c_{*}, \lambda\right)}-\int_{-\infty}^{0} u(\theta) e^{\lambda \theta} d \theta:=\frac{h(\lambda)}{\left(\lambda-\lambda_{*}\right)^{k+1}},
$$

where $k=1$ and

$$
h(\lambda)=\frac{\left(\lambda-\lambda_{*}\right)^{2} \int_{\mathbb{R}} e^{\lambda \theta} T(u)(\theta) d \theta}{\Delta\left(c_{*}, \lambda\right)}-\left(\lambda-\lambda_{*}\right)^{2} \int_{-\infty}^{0} u(\theta) e^{\lambda \theta} d \theta .
$$

By Lemma 2.1 (i), $\lambda_{*}$ is a double root of $\Delta\left(c_{*}, \lambda\right)=0$, and hence $\lim _{\lambda \rightarrow \lambda_{*}} h(\lambda)$ exists. Therefore, $h(\lambda)$ is analytic for all $0<\Re \lambda \leq \lambda_{*}$. Then Lemma 2.2 implies that

$$
\lim _{\xi \rightarrow+\infty} \frac{u(\xi)}{\xi e^{-\lambda_{*} \xi}} \text { exists; }
$$

that is,

$$
\lim _{\xi \rightarrow-\infty} \frac{\phi(\xi)}{|\xi| e^{\lambda_{*} \xi}} \text { exists. }
$$

Assume that $\phi(\xi)$ is not monotone for large $-\xi>0$. Letting $p=\frac{2 D+d}{c_{*}}$ and $\hat{\phi}(\xi)=\phi(\xi) e^{p \xi}>0$, it follows that

$c_{*} \hat{\phi}^{\prime}(\xi)=D\left[\hat{\phi}(\xi+1) e^{-p}+\hat{\phi}(\xi-1) e^{p}\right]+\left(c_{*} p-2 D-d\right) \phi(\xi)+b\left(\phi\left(\xi-c_{*} r\right)\right) e^{p \xi}>0$, which implies that $\hat{\phi}^{\prime}(\xi)>0$ for any $\xi \in \mathbb{R}$. Letting $\hat{u}(\xi)=\hat{\phi}(-\xi)$, it is obvious that $\hat{u}(\xi)$ is decreasing $\xi>0$. Let $\widehat{L}(\lambda)=\int_{-\infty}^{\infty} e^{-\lambda \xi} \hat{\phi}(\xi) d \xi$. Noting that $\widehat{L}(\lambda)=L(\lambda-p)$ and repeating the above argument, we have that

$$
\lim _{\xi \rightarrow+\infty} \frac{\hat{u}(\xi)}{\xi e^{-\left(p+\lambda^{*}\right) \xi}}=\lim _{\xi \rightarrow-\infty} \frac{\phi(\xi)}{|\xi| e^{\lambda^{*} \xi}} \text { exists. }
$$

This completes the proof.

Remark 2.1. Similarly to the proof of Theorem 2.1, without assuming the wave profile is monotone, we can also obtain that

$$
\lim _{\xi \rightarrow-\infty} \frac{\phi(\xi)}{e^{\lambda_{1} \xi}} \text { exists }
$$

for $c>c_{*}$, where $\lambda_{1}$ is given in Lemma 2.1 .

Theorem 2.2 (Uniqueness). Assume that (H1)-(H2) hold and that function $\beta$ has compact support. Let $\varphi, \psi$ be two traveling waves of (1.1) with the speed $c_{*}$. Then $\phi$ is a translation of $\psi$; more precisely, there exists $\bar{\xi} \in \mathbb{R}$ such that $\phi(\xi)=\psi(\xi+\bar{\xi})$.

Proof. Let $\varphi, \psi$ be two traveling waves for $c=c_{*}$. According to Theorem 2.1, there exist some positive numbers $\theta_{1}$ and $\theta_{2}$ such that

$$
\lim _{\xi \rightarrow-\infty} \frac{\phi(\xi)}{|\xi| e^{\lambda_{*} \xi}}=\theta_{1} \text { and } \lim _{\xi \rightarrow-\infty} \frac{\psi(\xi)}{|\xi| e^{\lambda_{*} \xi}}=\theta_{2} .
$$

Define

$$
w_{\epsilon}(\xi):=\frac{\phi(\xi)-\psi(\xi+\bar{\xi})}{(\varepsilon|\xi|+1) e^{\lambda_{*} \xi}}
$$


where $\bar{\xi}=\frac{1}{\lambda_{*}} \ln \frac{\theta_{1}}{\theta_{2}}$. Then $w_{\varepsilon}( \pm \infty)=0$, and hence $\max _{\xi \in \mathbb{R}}\left\{w_{\epsilon}(\xi)\right\}$ and $\min _{\xi \in \mathbb{R}}\left\{w_{\epsilon}(\xi)\right\}$ exist. Without loss of generality, we assume $\max _{\xi \in \mathbb{R}}\left\{w_{\epsilon}(\xi)\right\} \geq\left|\min _{\xi \in \mathbb{R}}\left\{w_{\epsilon}(\xi)\right\}\right|$ (otherwise, we may consider $\left.w_{\epsilon}(\xi):=\frac{\psi(\xi+\bar{\xi})-\phi(\xi)}{(\varepsilon|\xi|+1) e^{\lambda_{*} \xi}}\right)$. So, if $w_{\epsilon}(\xi) \not \equiv 0$, there exists $\xi_{0}^{\epsilon}$ such that

$$
w_{\epsilon}\left(\xi_{0}^{\epsilon}\right)=\max _{\xi \in \mathbb{R}}\left\{w_{\epsilon}(\xi)\right\}>0 \text { and } w_{\epsilon}^{\prime}\left(\xi_{0}\right)=0 .
$$

We first suppose that $\xi_{0}^{\epsilon} \rightarrow \infty$ as $\epsilon \rightarrow 0$. Choose $\epsilon>0$ sufficiently small such that $\xi_{0}^{\epsilon}>\sup \{j: j \in \operatorname{supp} \beta(j)\}+\max \left\{1, c_{*} r\right\}$. Note that

$$
\phi^{\prime}\left(\xi_{0}^{\epsilon}\right)-\psi^{\prime}\left(\xi_{0}^{\epsilon}+\bar{\xi}\right)=w_{\epsilon}^{\prime}\left(\xi_{0}^{\epsilon}\right)\left(\epsilon \xi_{0}^{\epsilon}+1\right) e^{\lambda_{*} \xi}+w_{\epsilon}\left(\xi_{0}^{\epsilon}\right) \epsilon e^{\lambda_{*} \xi}+w_{\epsilon}\left(\xi_{0}^{\epsilon}\right)\left(\epsilon \xi_{0}^{\epsilon}+1\right) \lambda_{*} e^{\lambda_{*} \xi},
$$

and for all $u, v \geq 0$,

$$
|b(u)-b(v)| \leq b^{\prime}(0)|u-v|
$$

Thus, we have

$$
\begin{aligned}
& c_{*} w_{\epsilon}\left(\xi_{0}^{\epsilon}\right) \epsilon e^{\lambda_{*} \xi}+c_{*} w_{\epsilon}\left(\xi_{0}^{\epsilon}\right)\left(\epsilon \xi_{0}^{\epsilon}+1\right) \lambda_{*} e^{\lambda_{*} \xi} \\
= & D\left\{\left[\phi\left(\xi_{0}^{\epsilon}+1\right)-\psi\left(\xi_{0}^{\epsilon}+\bar{\xi}+1\right)\right]+\left[\phi\left(\xi_{0}^{\epsilon}-1\right)-\psi\left(\xi_{0}^{\epsilon}+\bar{\xi}-1\right)\right]\right. \\
& \left.-2\left[\phi\left(\xi_{0}^{\epsilon}\right)-\psi\left(\xi_{0}^{\epsilon}+\bar{\xi}\right)\right]\right\}-d\left[\phi\left(\xi_{0}^{\epsilon}\right)-\psi\left(\xi_{0}^{\epsilon}+\bar{\xi}\right)\right] \\
& +\sum_{j \in \mathbb{Z}} \beta(j)\left[b\left(\phi\left(\xi_{0}^{\epsilon}-j-c_{*} r\right)\right)-b\left(\psi\left(\xi_{0}^{\epsilon}+\bar{\xi}-j-c_{*} r\right)\right)\right] \\
\leq & D\left\{w\left(\xi_{0}^{\epsilon}+1\right)\left[\epsilon\left(\xi_{0}^{\epsilon}+1\right)+1\right] e^{\lambda_{*}\left(\xi_{0}^{\epsilon}+1\right)}+w\left(\xi_{0}^{\epsilon}-1\right)\left[\epsilon\left(\xi_{0}^{\epsilon}-1\right)+1\right] e^{\lambda_{*}\left(\xi_{0}^{\epsilon}-1\right)}\right. \\
& \left.-2 w\left(\xi_{0}^{\epsilon}\right)\left(\epsilon \xi_{0}^{\epsilon}+1\right) e^{\lambda_{*} \xi_{0}^{\epsilon}}\right\}-d w\left(\xi_{0}^{\epsilon}\right)\left(\epsilon \xi_{0}^{\epsilon}+1\right) e^{\lambda_{*} \xi_{0}^{\epsilon}} \\
& +b^{\prime}(0) \sum_{j \in \mathbb{Z}} \beta(j)\left|w\left(\xi_{0}^{\epsilon}-j-c_{*} r\right)\right|\left[\epsilon\left(\xi_{0}^{\epsilon}-j-c_{*} r\right)+1\right] e^{\lambda_{*}\left(\xi_{0}^{\epsilon}-j-c_{*} r\right)} .
\end{aligned}
$$

It follows from (2.5), $\Delta\left(c_{*}, \lambda_{*}\right)=0$ and

$$
c_{*}=D\left(e^{\lambda_{*}}-e^{-\lambda_{*}}\right)-b^{\prime}(0) \sum_{j \in \mathbb{Z}} \beta(j)\left(j+c_{*} r\right) e^{-\lambda_{*}\left(j+c_{*} r\right)}
$$

that

$$
w_{\epsilon}\left(\xi_{0}^{\epsilon}\right)=w_{\epsilon}\left(\xi_{0}^{\epsilon} \pm 1\right) .
$$

Indeed, assume that $w_{\epsilon}\left(\xi_{0}^{\epsilon}\right)>w_{\epsilon}\left(\xi_{0}^{\epsilon}+1\right)$ or $w_{\epsilon}\left(\xi_{0}^{\epsilon}\right)>w_{\epsilon}\left(\xi_{0}^{\epsilon}-1\right)$. Then

$$
\begin{aligned}
& c_{*} w_{\epsilon}\left(\xi_{0}^{\epsilon}\right) \epsilon e^{\lambda_{*} \xi}+c_{*} w_{\epsilon}\left(\xi_{0}^{\epsilon}\right)\left(\epsilon \xi_{0}^{\epsilon}+1\right) \lambda_{*} e^{\lambda_{*} \xi} \\
< & D\left\{w_{\epsilon}\left(\xi_{0}^{\epsilon}\right)\left[\epsilon\left(\xi_{0}^{\epsilon}+1\right)+1\right] e^{\lambda_{*}\left(\xi_{0}^{\epsilon}+1\right)}+w_{\epsilon}\left(\xi_{0}^{\epsilon}\right)\left[\epsilon\left(\xi_{0}^{\epsilon}-1\right)+1\right] e^{\lambda_{*}\left(\xi_{0}^{\epsilon}-1\right)}\right. \\
& \left.-2 w\left(\xi_{0}^{\epsilon}\right)\left(\epsilon \xi_{0}^{\epsilon}+1\right) e^{\lambda_{*} \xi_{0}^{\epsilon}}\right\}-d w\left(\xi_{0}^{\epsilon}\right)\left(\epsilon \xi_{0}^{\epsilon}+1\right) e^{\lambda_{*} \xi_{0}^{\epsilon}} \\
& +b^{\prime}(0) \sum_{j \in \mathbb{Z}} \beta(j) w_{\epsilon}\left(\xi_{0}^{\epsilon}\right)\left[\epsilon\left(\xi_{0}^{\epsilon}-j-c_{*} r\right)+1\right] e^{\lambda_{*}\left(\xi_{0}^{\epsilon}-j-c_{*} r\right)},
\end{aligned}
$$

which implies that

$$
c_{*}<D\left(e^{\lambda_{*}}-e^{-\lambda_{*}}\right)-b^{\prime}(0) \sum_{j \in \mathbb{Z}} \beta(j)\left(j+c_{*} r\right) e^{-\lambda_{*}\left(j+c_{*} r\right)} .
$$

This is a contradiction to (2.6). By the bootstrapping arguments, we have $w_{\epsilon}\left(\xi_{0}^{\epsilon}\right)=$ $w_{\epsilon}\left(\xi_{0}^{\epsilon}-j\right)$ for $j \in \mathbb{Z}$, which implies that $w_{\epsilon}$ is a constant. Since $w_{\epsilon}(+\infty)=0$, we get $\phi \equiv \psi$. 
Next we assume that $\xi_{0}^{\epsilon} \rightarrow-\infty$ as $\epsilon \rightarrow 0$. Then $w_{\epsilon}\left(\xi_{0}^{\epsilon}\right) \rightarrow 0$, as $\epsilon \rightarrow 0$. Since

$$
\lim _{\epsilon \rightarrow 0} w_{\epsilon}(\xi)=w(\xi):=\frac{\phi(\xi)-\psi(\xi+\bar{\xi})}{e^{\lambda_{*} \xi}} \text { for all } \xi \in \mathbb{R}
$$

and $w_{\epsilon}(x) \leq w_{\epsilon}\left(\xi_{0}^{\epsilon}\right)$, we have $w(\xi) \leq 0$ for all $\xi \in \mathbb{R}$. Note that $w_{\epsilon}\left(\xi_{0}^{\epsilon}\right)>0$ implies $\phi\left(\xi_{0}^{\epsilon}\right)-\psi\left(\xi_{0}^{\epsilon}+\bar{\xi}\right)>0$ and hence $w\left(\xi_{0}^{\epsilon}\right)>0$, which gives a contradiction.

Lastly, we assume $\left\{\xi_{0}^{\epsilon}\right\}$ is bounded. Then we can take a subsequence $\xi_{0}^{\epsilon} \rightarrow \xi_{1}$ as $\epsilon \rightarrow 0$, for some finite $\xi_{1}$. From uniform convergence of $w_{\epsilon}$ to $w$ on compact sets, $w_{\epsilon}\left(\xi_{0}^{\epsilon}\right) \rightarrow w\left(\xi_{1}\right)$ as $\epsilon \rightarrow 0$, where $w(\xi)$ is defined by (2.8). Thus, $w(\xi)=$ $\lim _{\epsilon \rightarrow 0} w_{\epsilon}(\xi) \leq \lim _{\epsilon \rightarrow 0} w_{\epsilon}\left(\xi_{0}^{\epsilon}\right)=w\left(\xi_{1}\right)$ for all $\xi \in \mathbb{R}$. Now we begin with repeating the above argument for $w(\xi)$. It is obvious that $w\left(\xi_{1}\right)=\max _{\xi \in \mathbb{R}}\{w(\xi)\} \geq 0$ and $w^{\prime}\left(\xi_{1}\right)=0$. Since $\max _{\xi \in \mathbb{R}}\left\{w_{\epsilon}(\xi)\right\} \geq\left|\min _{\xi \in \mathbb{R}}\left\{w_{\epsilon}(\xi)\right\}\right|$, we have $\max _{\xi \in \mathbb{R}}\{w(\xi)\} \geq\left|\min _{\xi \in \mathbb{R}}\{w(\xi)\}\right|$. We claim that $w\left(\xi_{1}\right)=w\left(\xi_{1} \pm 1\right)$. Assume, for the sake of contradiction, that either $w\left(\xi_{1}+1\right)<w\left(\xi_{1}\right)$ or $w\left(\xi_{1}-1\right)<w\left(\xi_{1}\right)$. According to (2.4) and $\phi^{\prime}\left(\xi_{1}\right)-\psi^{\prime}\left(\xi_{1}+\bar{\xi}\right)=$ $w^{\prime}\left(\xi_{1}\right) e^{\lambda_{*} \xi_{1}}+\lambda_{*} w\left(\xi_{1}\right) e^{\lambda_{*} \xi_{1}}$, we obtain

$$
\begin{aligned}
c_{*} \lambda_{*} w\left(\xi_{1}\right) e^{\lambda_{*} \xi_{1}}= & D\left[w\left(\xi_{1}+1\right) e^{\lambda_{*}\left(\xi_{1}+1\right)}+w\left(\xi_{1}-1\right) e^{\lambda_{*}\left(\xi_{1}-1\right)}-2 w\left(\xi_{1}\right) e^{\lambda_{*} \xi_{1}}\right] \\
& -d w\left(\xi_{1}\right) e^{\lambda_{*} \xi_{1}}+\sum_{j \in \mathbb{Z}} \beta(j)\left[b\left(\phi\left(\xi_{0}^{\epsilon}-j-c_{*} r\right)\right)-b\left(\psi\left(\xi_{0}^{\epsilon}+\bar{\xi}-j-c_{*} r\right)\right)\right] \\
\leq & {\left[w\left(\xi_{1}+1\right) e^{\lambda_{*}\left(\xi_{1}+1\right)}+w\left(\xi_{1}-1\right) e^{\lambda_{*}\left(\xi_{1}-1\right)}-2 w\left(\xi_{1}\right) e^{\lambda_{*} \xi_{1}}\right] } \\
& -d w\left(\xi_{1}\right) e^{\lambda_{*} \xi_{1}}+b^{\prime}(0) \sum_{j \in \mathbb{Z}} \beta(j)\left|w\left(\xi_{1}-j-c_{*} r\right)\right| e^{\lambda_{*}\left(\xi_{1}-j-c_{*} r\right)} \\
< & {\left[w\left(\xi_{1}\right) e^{\lambda_{*}\left(\xi_{1}+1\right)}+w\left(\xi_{1}\right) e^{\lambda_{*}\left(\xi_{1}-1\right)}-2 w\left(\xi_{1}\right) e^{\lambda_{*} \xi_{1}}\right] } \\
& -d w\left(\xi_{1}\right) e^{\lambda_{*} \xi_{1}}+b^{\prime}(0) \sum_{j \in \mathbb{Z}} \beta(j) w\left(\xi_{1}\right) e^{\lambda_{*}\left(\xi_{1}-j-c_{*} r\right)},
\end{aligned}
$$

which is a contradiction to $\Delta\left(c_{*}, \lambda_{*}\right)=0$. Repeating the above argument and by $w(+\infty)=0$, we have $\phi \equiv \psi$. This completes the proof.

\section{ACKNOWLEDGEMENTS}

The author would like to thank the referee and the editor for valuable comments and helpful suggestions which led to an improvement of the original manuscript.

\section{REFERENCES}

1. M. Aguerrea, S. Trofimchuk, G. Valenzuela, Uniqueness of fast travelling fronts in reactiondiffusion equations with delay, Proc. Roy. Soc. A, 464 (2008), 2591-2608. MR 2439284 (2010f:35167)

2. J. Carr, A. Chmaj, Uniqueness of travelling waves for nonlocal monostable equations, Proc. Amer. Math. Soc., 132 (2004), 2433-2439. MR2052422 (2005b:35125)

3. X. Chen, S. Fu, J. Guo, Uniqueness and asymptotics of traveling waves of monostable dynamics on lattices, SIAM J. Math. Anal., 38 (2006), 233-258. MR2217316 (2007i:37154)

4. X. Chen, J. Guo, Uniqueness and existence of traveling waves for discrete quasilinear monostable dynamics, Math. Ann., 326 (2003), 123-146. MR.1981615(2004b:37175)

5. J. Coville, On uniqueness and monotonicity of solutions of nonlocal reaction diffusion equation, Ann. Mat. Pura Appl. (4), 185 (2006), 461-485. MR2231034(2007e:35156)

6. O. Diekmann, H.G. Kaper, On the bounded solutions of a nonlinear convolution equation, Nonlinear Anal. TMA, 2 (1978), 721-737. MR.512163 (80c:45015)

7. J. Fang, X. Zhao, Existence and uniqueness of traveling waves for non-monotone integral equations with applications, J. Differential Equations, 248 (2010), 2199-2226. MR2595719 (2011a:45019) 
8. J. Fang, J. Wei, X. Zhao, Spreading speeds and travelling waves for non-monotone timedelayed lattice equations, Proc. R. Soc. A, 466 (2010), 1919-1934. MR2652726

9. J. Fang, J. Wei, X. Zhao, Uniqueness of traveling waves for nonlocal lattice equations, Proc. Amer. Math. Soc., 139 (2011), no. 4, 1361-1373. MR2748428

10. S. Ma, Traveling waves for non-local delayed diffusion equations via auxiliary equations, J. Differential Equations, 237 (2007), 259-277. MR2330948 (2008f:35205)

11. S. Ma, J. Wu, Existence, uniqueness and asymptotic stability of traveling wavefronts in a non-local delayed diffusion equation, J. Dyn. Diff. Eqns, 19 (2007), 391-436. MR2333414 (2008f:35206)

12. S. Ma, X. Zou, Existence, uniqueness and stability of traveling waves in a discrete reactiondiffusion monostable equation with delay, J. Differential Equations, 217 (2005), 54-87. MR 2170528 (2006g:35277)

13. S. Ma, P. Weng, X. Zou, Asymptotic speed of propagation and traveling wavefronts in a non-local delayed lattice differential equation, Nonlinear Anal. TMA, 65 (2006), 1858-1890. MR 2258473(2007j:34162)

14. P. Weng, H. Huang, J. Wu, Asymptotic speed of propagation of wave fronts in a lattice delay differential equation with global interaction, IMA J. Appl. Math., 68 (2003), 409-439. MR:1988153 (2004c:37202)

College of Science, University of Shanghai for Science and Technology, Shanghai 200093, People's Republic of China

E-mail address: yzx3411422@163.com, yuzx0902@yahoo.com.cn 